\title{
Despeckling of SAR Images Using BEMD-Based Adaptive Frost Filter
}

\author{
Ranjith Kumar Painam ${ }^{1} \cdot$ M. Suchetha ${ }^{2}$
}

Received: 27 September 2021 / Accepted: 2 January 2022

(C) Indian Society of Remote Sensing 2022

\begin{abstract}
In image processing, removal of speckle noise from a satellite image is a challenging task for the researchers. There are various approaches for speckle noise reduction. Generally, the speckle noises are scattered, in satellite images, medical images and synthetic aperture radar (SAR) images. This paper introduces a bidimensional empirical mode decomposition (BEMD)-based adaptive filtering method for despeckling of SAR image. The noisy SAR image is decomposed into different bidimensional intrinsic mode function (BIMF) levels using BEMD and then filtering is performed on the first BIMF level, as it contains the high-frequency noise component. This adaptation process effectively filters out the noisy image component without destroying the original image component. A BEMD-based adaptive Frost filter is introduced in this paper for despeckling of SAR images. The despeckling performances of our proposed filtering method are further analyzed by visual evaluation and also using performance parameters comparatively. Our reconstructed images show better performance quantitatively and qualitatively compared to other filters.
\end{abstract}

Keywords Adaptive filters - BEMD $\cdot$ Synthetic aperture radar $\cdot$ Speckle noise $\cdot$ Image reconstruction

\section{Introduction}

Synthetic aperture radars (SARs) are used to produce highresolution images based on the principle of transmission and reception of echo signals $(\mathrm{Xu}, 1999)$. The physical properties of the immediate landscape $500 \mathrm{~m}$ from the residential location are described by the terrain category. Complete sheltering occurs when at least two groups of homes or other fixed impediments of equal weight encircle the building under consideration. Throughout broadcasting, this links the antennae to the broadcaster, while during receiving, it integrates toward the receivers. The signal received these brief pulses and transmits energy into spacecraft. The antenna has a high degree of directionality.

M. Suchetha

suchetha.m@vit.ac.in

Ranjith Kumar Painam

ranjithkumar.painam2015@vit.ac.in

1 School of Electronics Engineering, Centre for Healthcare Advancement, Innovation and Research, Vellore Institute of Technology, Chennai Campus, Chennai, Tamil Nadu, India

2 Centre for Healthcare Advancement, Innovation and Research, Vellore Institute of Technology, Chennai Campus, Chennai, Tamil Nadu, India
A feeble reflection sound rebounds to a certain antenna if the sent pulse reaches any object. The antennas are installed on mobile platforms like aircraft or spacecraft (Rott et al., 2010). For obtaining improved azimuth resolution and larger synthetic antenna, platform movement is used. The rotational breadth of the emitted narrow frequency band as well as achieving various lengths defines the azimuth located alongside precision. The breadth of the light distribution is measured by the beamwidth (A). Various applications of SAR include the mapping of terrain, sea surface, oil slick pollution monitoring, surface surveillance, mine detection, automatic target recognition and classification (Errico et al., 2015; Ramdani, 2019). Marine pollution includes oil slicks on the sea surface caused by spillage from boats, petroleum \& energy rigs, especially spontaneous seeping from the sea bottom. The amount of time required for the ultrasonic pulse to propagate among the higher and bottom edges of the oil slick may be used to calculate its depth. Terrain mapping and assessment are applied to detect earth characteristics in order to deduce their genesis and scientific understanding, as well as the types of earth materials elements that may be found in them, as well as their geological qualities and state. SAR images can be collected throughout the day even when there are stormy weather and heavy clouds 
(Coillie et al., 2011; Lu et al., 2014; Rajamani \& Krishnaveni, 2014). Automatically processing the SAR images was extremely difficult due to the speckle noise present in Khosravi et al. (2018), Zhang et al., (2013) and Warner and Lee (2011). SAR images are corrupted by speckle noise because of the constructive and destructive electromagnetic interference of waves during image collection. Speckle is a multiplicative noise which produces a grainy appearance to the image (Jidesh \& Balaji, 2018; Liu et al., 2017; Yuan et al., 2014). Even the commonly used processing algorithms like region classification or segmentation cannot give better performance if the input image is speckled (Idol et al., 2015). So it is essential to minimize the speckle noise in the primary preprocessing stage. The two different types of region-based segmentations are region splitting as well as merging technique and region developing technique.

\section{Related Works}

The literature exploration deals with various techniques of proposed annulment in SAR images. These include Lee filtering (Lee, 1980), Kuan filtering (Kuan et al., 1985), Frost filtering (Frost et al., 1982) and gamma MAP filtering (Hsiao et al., 2002). Lee and Kuan filters are adaptive mean filters, whereas a Frost filter is a mean adaptive weighted filter. A GMAP filter is introduced and it requires the probability density function (PDF) prior knowledge of the image before applying (Jia et al., 2019). The image reflectivity is considered to be a gamma distribution instead of a Gaussian distribution.

The gamma distribution is a 2 different class of continuum probability density functions used in statistical hypothesis testing. The gamma range includes specific examples such as the exponentially, Erlang, as well as chisquare distributions. The Gaussian distribution is a bellshaped arc in which numbers are supposed to test the normality including an equivalent number of data points along each total mean throughout every observation. It is otherwise called as normal distribution. A radar cross section (RCS) model for designing a speckle noise removal filter is introduced in Achim et al. (2006) and it is based on the heavy-tailed Rayleigh density function. The corresponding area observed by every radar is the target's radar cross section (RCS). Its the fictional region that intercepts the quantity of energy and, if dispersed evenly in all locations, generates a radar echo equivalent to the enemy targets. Bayesian filters are explained using the Bayesian theorem, which describes a posterior probability by a prior pdf. A Bayesian filter is a software that evaluates the header as well as substance of an arriving e-mail communication and determines the likelihood that it is junk using Bayesian reasoning, also known as Bayesian analysis.
Anti-virus tools should be utilized in combination with Bayesian filtering. The wavelet-based despeckling algorithms are proposed in Gleich and Datcu (2009) and Argenti et al., (2006), it is developed using MAP estimation and undecimated wavelet decomposition. Here, the assumption is that each wavelet coefficient's pdf is generalized Gaussian (GG). The parameters for GG pdf are used to obtain space-variation in every wavelet frame. Therefore, they can be modified to the context of the spatial image in addition to orientation and scale. The GARCH method is used to predict that may be used to examine a range of investment information, such as market indicators. This method is frequently applied organizations to predict the unpredictability of stocks, bond and macroeconomic indicators values. A 2-D GARCH model is used in despeckling of SAR images as presented in Amirmazlaghani et al. (2008), a logarithmic transformation of the original SAR image is decomposed into multi-scale wavelet domain. SAR images wavelet coefficients have got non-Gaussian statistics which are fully specified by this model. A sequential Monte Carlo method proposed in Gleich and Datcu (2009) is a model-based Bayesian approach. The second-generation wavelets such as bandelet (Lu et al., 2014), chirplet (Lian \& Jiang, 2017), contourlet (Metwalli et al., 2014) have been developed in the last few years. The process of despeckling the SAR images using contourlet transform (Li et al., 2006) and bandelet (Sveinsson et al., 2008) transform gives better despeckling results as compared with each wavelet-based method. The contourlet transform is a novel two-dimensional picture encoding transformation technique. The outlines of actual photos, which are the most prominent elements in environmental photos, may be managed to capture to use the wavelet transforms with just a few parameters. The bandelet is a multiresolution linear transformation that maintains the geometrical integrity of pictures and materials. The bandelet transformation takes use of the mathematical consistency of a picture's architecture and is useful for analyzing picture borders and texturing. In the wavelet domain, the noise and image models have defined the results in Gleich and Datcu (2009) and Argenti and Alparone (2002) using MAP estimation of the denoised image evaluated. A maximum a posteriori probability (MAP) estimation in Bayesian inference is an estimation of an arbitrary function that matches the subsequent distribution's feature. Mostly on analysis of the experimental information, the MAP may be used to produce a prediction equation of a multitude of settings. The speckle noise is a type of multiplicative noise which is present in the SAR image (Yuan et al., 2014). Speckle is a granular interference that occurs naturally in dynamic radiation, synthetic aperture radar (SAR), clinical ultrasonography and optically coherent scanning pictures and decreases their clarity. 
Coordinated reception of diffracted information from several dispersed sources is the reason. The RADARSAT-2 dataset was subjected toward the speckled filtration, which included modified Lee, boxcar, enhanced Lee-Sigma and IDAN filtration. Speckle filtering must reduce random noise while maintaining spatially and analysis required data. Hybrid polarimetric information is used to assess speckled filtering. Based on other wavelet denoising techniques (Liu et al., 2017) use logarithmic transform to change the multiplicative noise model into an additive noise model (Achim et al., 2006). The logarithmically modified image is designed by employing filtering methods like zero-mean Gaussian distributions and zero location Cauchy approaches (Bhuiyan et al., 2007). This is done to generate the MAP estimator and minimum mean absolute error estimator. The extension of the Kalman filter is the wiener filter. A recursive unscented Kalman filter (UKF) was presented in Subrahmanyam et al. (2008) and this does not require any parameter estimation to incorporate nonGaussian prior. The unscented Kalman filter is a substandard nonlinear filtering technique that, unlike EKF or LKF, employs an unprocessed transition (UT) instead of exponential function to linearize nonlinear solutions. The Kalman filter algorithm employs a dynamical framework to explain an angular velocity and is the best approach for estimating its condition if the program's modeling is straight or the procedure and inaccurate data are cumulative and stochastic. The well-known algorithm is the extended Kalman filter, and it provides a nonlinear and non-Gaussian model, it depends on the principle of linearization of the evolution as well as measurement models utilizing the Taylor series. The particle filtering (Gleich \& Datcu, 2009) method presents a solution for solving the nonlinear filtering and non-Gaussian problems, which are having numerical Bayesian techniques. For solving tracking problems (Godsill \& Clapp, 2001), particle filters were adopted. Recently, it is seen applied for resolving the problems related to source separation (Everson \& Roberts, 2000), object collision (Tamminen \& Lampinen, 2006), segmentation (Li et al., 2006) and road detection (Chen et al., 2006). Kalman filter can be applied for despeckling the SAR images (Geling \& Ionescu, 1994), but the filter parameters will modify the SAR image local area. Using the Kalman filter, the segmented regions of SAR images are despeckled (Tsuchida et al., 2003). A two-dimensional adaptive block Kalman filter process is presented in AzimiSadjadi and Bannour (1991). Despeckling of SAR image with a particle filter is proposed in Gencaga et al. (2005). Despeckling of SAR image using total variational (TV) models presented in Woo and Yun (2011), an alternating minimization algorithm with shift technique (AMAST) algorithm is used to remove the speckle noise, and it is based on the Lagrangian function. A linearized proximal alternating minimization algorithm (LPAMA) (Yun \& Woo, 2012) is used to reduce the multiplicative noise in SAR images. It is an ' $m$ 'th root transformed total variational (TV) model. Two despeckling models presented in Feng et al. (2014) based upon the total generalized variation (TGV) regularization. These two models are developed based on an algorithm called prima dual method (PD) with TGV penalty (PDTGV). The first model is called PDTGV exponential model and the second model is called PDTGV I-divergence model. PDTGVs can obtain smooth regions and discontinuities at the object boundaries. PDTGVs remove the staircasing artifacts produced by the TV (Ahmed et al., 2012; Balamurugan et al., 2019; Nguyen et al., 2018). Based on TV algorithms, bring staircasing artifacts in which that are absent when working with PDTGV exponential model. PDTGV I-divergence model removes any inverse operations or inner iterations, whereby this obtains a considerable speed advantage. A Gaussian denoising-based multi-channel SAR image speckle removal technique called MuLoG (MUlti-channel Logarithm) is presented (Deledalle et al., 2017), and it is used in a speckle reduction filter. MuLoG is helpful to remove structures appearing only with single Gaussian denoiser as artifacts.

Even though many filtering methods are emphasized in the literature, it lacks in the discussion on adaptivity based on noise variations and spatial domain filtering approaches. Our proposed method is focused on adaptiveness based on noise components in various levels and also filtering in the spatial domain. The major contributions in our detailed analysis of proposed work are projected below:

(a) The decomposition of original and noisy images is done to analyze the characteristics of speckle noise in a SAR image. This is experimentally done by analyzing the frequency components in each BIMF level.

(b) We have proposed a hybridized adaptive filtering method with BEMD-based Frost filter.

(c) Detailed performance analysis has been carried out between the proposed filtering methods and the existing despeckling methods. This is done in two ways (i) visual assessment and (ii) using performance parameters.

(d) The analytical and experimental results are comparatively studied with varying filter window sizes and also at various variances of noise.

This paper is organized as follows. In "Theoretical Background" section, we are explaining the theoretical background of BEMD and adaptive filters. In "Proposed Methodology" section, the proposed methodology is explored. In "Results and Discussion" section, we are comparing the conventional and proposed BEMD-based 
adaptive filters, the study and analysis have been concluded. The effectiveness of proposed methodology is evaluated by various performance parameters.

\section{Theoretical Background}

\section{Speckle Noise Model in SAR Image}

A SAR image can be mathematically written as

$U\left(i_{o}, j_{o}\right)=V\left(i_{o}, j_{o}\right) \cdot S_{n}\left(i_{o}, j_{o}\right)$

where $\left(i_{o}, j_{o}\right)$ is the azimuth coordinates and spatial range of the resolution cell around the center, $U\left(i_{o}, j_{o}\right)$ indicates SAR image intensity and it is a random process of radar reflectivity(unspeckled radiance), $S_{n}\left(i_{o}, j_{o}\right)$ is modeled as a stationary random process with speckle noise having unit variance and mean. This is how the Altitude-Azimuth (else Alt-Az) reference method operates, allocating degrees to lateral and vertical motions. The elevation of an item over the horizons, defined as an inclination, is referred to as altitude. The organism's elevation is 0 degrees if someone is on the ground. This is in proportion to the effective number of looks $\mathrm{N}$ and it is statistically independent of $V\left(i_{o}, j_{o}\right)$.

\section{BEMD Algorithm}

Linderhed (Linderhed, 2002) introduced the BEMD concept. The spatial information of the image pixel can be obtained using BEMD. BEMD (bidimensional empirical mode decomposition) is a new degradation approach that relies on oscillating feature data collecting. The BEMD approach is used to analyze non-stationary as well as nonlinear signals. Internal mode parameters are dynamically reduced signals into inherent oscillation elements. An imperative advance in EMD procedure involves the development of maxima and minima envelopes. Further to the investigation, it is proved that the most appropriate fit for connecting the extrema is the cubic spline. The implementation steps for BEMD are explained as Algorithm 1.

\section{BEMD Algorithm}

Input image $X_{1}(p, q)$ the following steps are proceeded.

Step 1 Identify all local maxima and local minima of the SAR input image $X_{1}(p, q)$.

Step 2 Compute the upper envelope $E_{\max }(p, q)$ and lower envelope $E_{\min }(p, q)$.

Step 3 Connect the upper envelope and lower envelope by 2 -D cubic spline.

Step 4 Determine the mean for every spatial position
$E M_{m}^{n}(p, q)=\frac{E_{\max }(p, q)+E_{\min }(p, q)}{2}$

where $n$ is the iteration number, $m=1,2,3, \ldots M$, where $M$ represents the number of BIMFs.

Step 5 Subtract the mean envelope from the input image $S_{m}^{n}(p, q)=X_{1, m}^{n}(p, q)-E M_{m}^{n}(p, q)$.

This corresponds to one iteration and the process is called as sifting.

Step 6 Determine whether the resultant image is a BIMF from step 5. The stopping criterion for the process checking is an envelope mean that is to be close to zero.

$\frac{\sum_{p=l}^{P} \sum_{q=l}^{R}\left|E M_{m}^{n}(p, q)\right|}{P_{x} R}<\tau$

where $P$ and $R$ are the dimensions of the envelope, $\tau$ represents the threshold value which is almost equal to zero. If the stopping criterion is fulfilled at $N$ th $(n=N)$ iteration, then the current BIMF can be formulated as

$\operatorname{BIMF}_{1, m}(p, q)=S_{m}^{N}(p, q)$

check the resultant is a BIMF, otherwise continue the process from step 1 till finding the first BIMF

$I_{1, m}^{n+1}(p, q)=S_{m}^{n+1}(p, q)$.

Step 7 If the current BIMF is obtained successfully, the residue image

$R_{m}(p, q)=I_{l, m}^{n}(p, q)-\operatorname{BIMF}_{l, m}(p, q)$ is determined.

Step 8 Finally original image can be written as

$X_{1}(p, q)=\sum_{m=1}^{M} \mathrm{BIMF}_{l, m}(p, q)+R_{m}(p, q)$.

\section{2-D Adaptive Filters}

Adaptive filters are commonly applied in image processing to enhance the image by removing the noise without significantly blurring the structures in the image (AzimiSadjadi \& Pan, 1994). An adaptive filter is a device with such a modulation technique and an integral gain governed through control factors that may be adjusted using an evaluation method. Mostly all filter methods are digitized filtration owing to the complicated of the techniques. Figure 1 shows the architecture of a two-dimensional adaptive filter. The noisy image $X_{i, j}(k, l)$ is given as input to the adaptive filter and the reference image $D_{i, j}(k, l)$ is given to perform adaptation. The 2-D adaptive filter makes an iterative adaptation, and the resultant image $Y_{i, j}(k, l)$ is obtained. The mean square error is used in this process to 


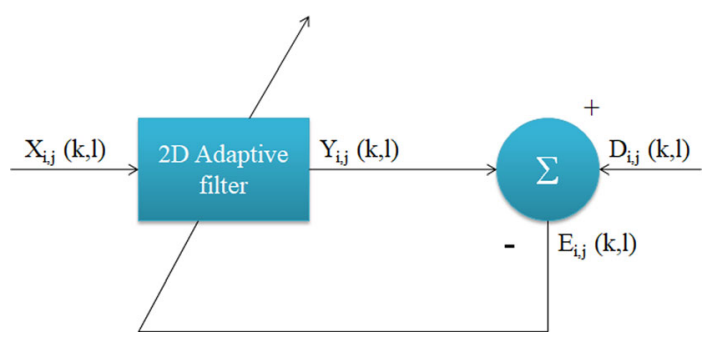

Fig. 1: 2-D adaptive filter architecture

measure the difference between the estimated image and the reference image.

\section{Proposed Methodology}

\section{Database Collection}

We have collected SAR images from Sandia National Laboratory using the following link. http://www.sandia. gov/radar/complex-data/. This database has a collection of several SAR images with different sizes, and each image has azimuth and range resolution. The SAR images are of size $3000 \times 1754$, azimuth resolution $(\mathrm{m})$ is 0.12000251 and range resolution (m) is 0.11997993 .

\section{BEMD-Based Adaptive Filtering}

The use of EMD for 2-D data is termed as bidimensional empirical mode decomposition (BEMD) (Nunes \& Deléchelle, 2009). Our proposed adaptive filtering process using BEMD is shown in Fig. 2.

Noisy SAR image is initially decomposed into various levels of BIMF using the decomposition process called sifting. This process depends on two constraints, (i) every BIMF should maintain a similar number of zero crossings and extrema and (ii) the local mean of every BIMF should be symmetric. Here, we are selecting the first BIMF because the noise concentration is more on the higher frequency component of the image. Thus the first BIMF level is adaptively filtered. The output of the adaptively filtered image is combined with the remaining BIMFs and residue. Finally, the output of the adder is a despeckled SAR image. The effect of speckle noise can be effectively reduced using BEMD algorithm combined with adaptive filtering.

\section{Proposed BEMD-Based Frost Filter}

The proposed BEMD-based Frost filter uses the first BIMF obtained after performing BEMD decomposition on input image. This filter is derived based on the minimum mean square error (MMSE) criteria for smoothing the SAR images (Frost et al., 1982). In statistical and communication systems, a minimal mean square error (MMSE) predictor is an estimate technique that minimizes the mean square error (MSE) of the estimated coefficients of a regression model, which is a typical measure of estimating performance. For retaining the edge structures of the image, the BEMD-based Frost filter will compute the locally determined parameter values and will give the MMSE estimation within the homogeneous regions. It uses a negative exponential distribution, and for filtering process, this filter uses local image statistics. Thus by local image statistics, the BEMD-based Frost filter can able to give weights of each cell within the filter window. The cell Weight is always depending on the distance calculated from the center of the cell.
Fig. 2 Block diagram of proposed BEMD-based adaptive filtering process

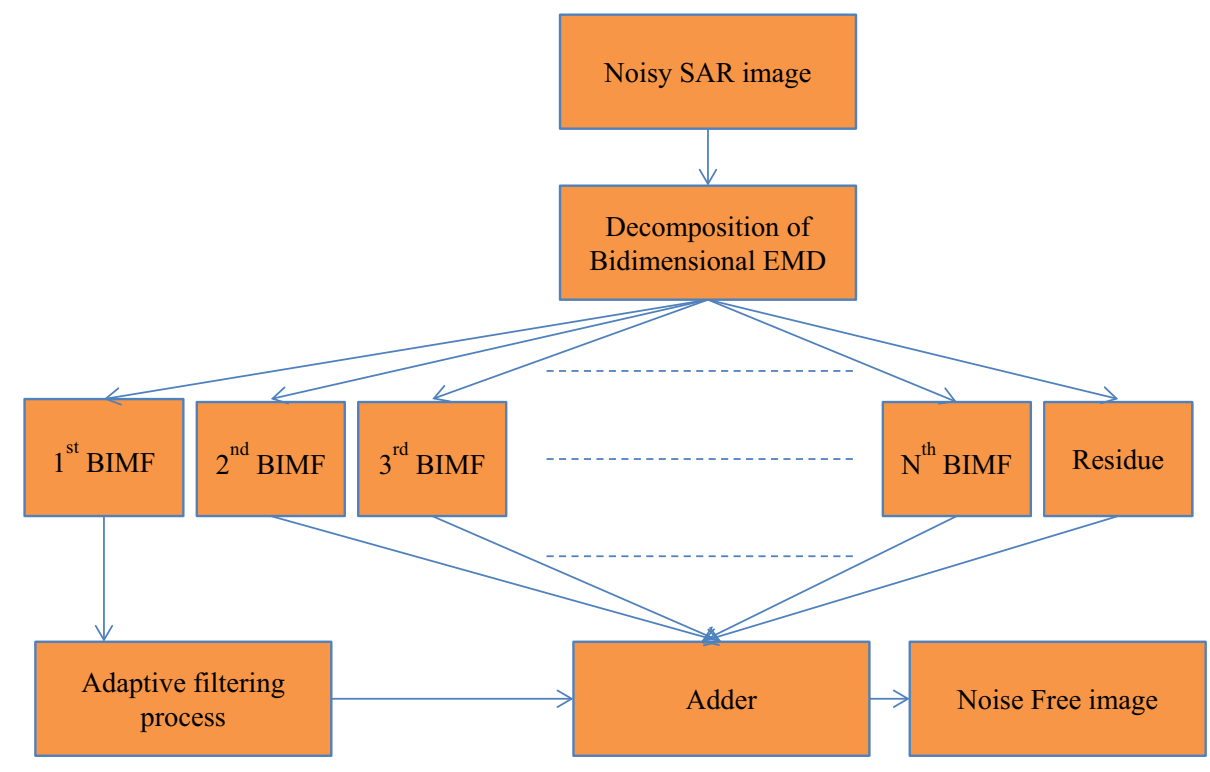




\section{Development of BEMD-Based Frost Filter}

The proposed filtering model is developed using the MMSE criterion to obtain the functional form of the filter (Domg \& Milne, 2001). The Frost filter preserves the edges while reducing the distortion in a sliding window depending on specific data. The Damping element, which is also an exponentially dampening, is the most important component in determining the filter's smoothing. Whenever the damping factor is low, the picture is clear. Then, $f(p, q)$ is estimated from $I(p, q)$ under the stationary assumptions of image data. The impulse response $m(t)$ and the transfer function $M(r)$ of the MMSE filter gives an estimate of $f(p, q)$ from $I(p, q)$, and it is represented by minimizing the mean square error $\epsilon^{2}$ given by

$\epsilon^{2}=E\left[(f(t)-I(t) * m(t))^{2}\right]$

the above equation is replaced with $t=(p, q)$ spatial coordinates. The MMSE solution leads to a transfer function as

$M(r)= \begin{cases}{\left[\frac{\bar{n} S_{f}(r)}{S_{f}(r) * S_{n}(r)}\right] \frac{1}{H^{*}(r)},} & r \neq 0 \\ \frac{1}{\bar{n}}, & r=0\end{cases}$

where $\bar{n}=E[n(t)]$ and $r=\left(r_{i}, r_{j}\right)$ are the spatial frequency coordinates. $S_{n}(r)$ is the noise process and $S_{f}(r)$ is the power spectral density. $H^{*}(r)$ is the complex conjugate of the transfer function of the system and it can be treated as a constant. The remaining part of $M^{\prime}(r)$ is $\frac{\bar{n} S_{f}(r)}{S_{f}(r) * S_{n}(r)}$ and it can be considered as a data dependent. The standard model for $f(t)$ is an autoregressive process with an autocorrelation function $R_{f}(\tau)$ and a two-sided power spectral density $S_{f}(r)$ of the form (Habibi, 1972),

$R_{f}(\tau)=\sigma_{f}^{2} e^{-a(\tau)}+\bar{f}^{2}$

where the parameter $\sigma_{f}^{2}, \bar{f}^{2}$ and $a$ have different values for different terrain categories. The physical properties of the immediate landscape $500 \mathrm{~m}$ from the residential location are described by the terrain category. Complete sheltering occurs when at least two groups of homes or other fixed impediments of equal weight encircle the building under consideration.

$S_{f}(r)=\frac{2 \sigma_{f}^{2} a}{a^{2}+4 \pi^{2} r^{2}}+\bar{f}^{2} \delta(r)$.

The correlation $R_{n}(\tau)$ and power spectral density $S_{f}(r)$ for the multiplicative white noise are

$R_{n}(\tau)=\sigma_{n}^{2} \delta(\tau)+\bar{n}^{2}$
$S_{n}(r)=\sigma_{n}^{2}+\bar{n}^{2} \delta(r)$

where the parameters $\sigma_{n}^{2}$ and $\bar{n}^{2}$ are the variance of SAR image and the number of looks, respectively. With the power spectral densities of $f(t)$ and $n(t)$, we can deduce the impulse response of the filter as (Frost et al., 1980)

$m^{\prime}(t)=K_{1} \alpha e^{-\alpha|t|}$

and

$\alpha=\sqrt{2 a\left[\frac{\bar{n}}{\sigma_{n}}\right]^{2} \cdot\left[\frac{1}{1+\left(\frac{\bar{f}}{\sigma_{f}}\right)^{2}}\right]+a}$

where the decay constant, $\alpha$, is a function of all three signal parameters $\sigma_{f}, \bar{f}$ and $a$. The homogeneous area $a$ will change by a small amount. So $a$ can be treated as a constant for the purpose of adaptiveness in the filter.

\section{Implementation of Proposed BEMD-Based Frost Filter}

The steps for implementation of BEMD-based Frost filter are discussed as Algorithm 2. The noisy image is decomposed using BEMD. This filter works like mean filter while uniform regions are filtered and work as a high pass filter while high contrast regions are filtered (Lopes et al., 1990; Zhu et al., 2013). So, the high contrast regions will be preserved, and the uniform regions will be smoothened by eliminating the speckle noise. In the BEMD-based Frost filter, the image is relatively approximated by convolving the observed image along with the impulse response of the SAR system.

\section{Proposed BEMD-Based Frost Filter Algorithm}

Step 1 Decompose noisy image using BEMD to get $\operatorname{BIMF}_{l, m}(p, q)$.

Step 2 Take first $\mathrm{BIMF}_{l, m}(p, q)$.

Step 3 Apply BEMD-based Frost filter on $\operatorname{BIMF}_{l, m}(p, q)$ image.

Step 4 Apply a window size region centered around the pixel $(i, j)$.

Step 5 Calculate

$$
I\left(p_{o}, q_{o}\right)=\sum_{p=-k}^{k} \sum_{q=-k}^{k} m\left(x_{o}+p, y_{o}+q\right) I\left(x_{o}+p, y_{o}+q\right)
$$

.where $(2 k+1) x(2 k+1)$ is the size of window $m(x+p, y+q)=K_{1} \alpha \exp (-\alpha t), t=\sqrt{p^{2}+q^{2}}$ and $\alpha$ is computed using the following equation 
$\alpha=\sqrt{2 a\left[\frac{\bar{n}}{\sigma_{n}}\right]^{2} \cdot\left[\frac{1}{1+\left(\frac{\bar{f}}{\sigma_{f}}\right)^{2}}\right]+a}$.

Step 6 Replace the value of the center pixel with the value $I(p, q)$.

Step 7 Proceed to the next pixel in the SAR image and repeat from step 1 till the entire image is processed.

\section{Results and Discussion}

In this paper, we have used BEMD-based adaptive filtering methods to minimize the noise level. The collected SAR images from the database of Sandia National Laboratory are first decomposed into various BIMFs. Further, a spatial domain adaptive filter is applied to the noisy BIMF level (BIMF-1). This decomposition-based filter hybridization results in better adaptation in minimizing the speckle noise in images. The experimental and analysis results are presented in this section.

The evaluation of SAR images after BEMD-based adaptive filtering process is carried out in two ways, (i) visual evaluation and (i) calculation of performance parameters. Figure 3 shows the BEMD decomposition of a SAR image. Figure $3 \mathrm{a}$ shows our original SAR image. A SAR image with speckle noise 0.05 is shown in Fig. 3b, and Fig. 3c-e shows the three BIMF levels. Figure 3c shows the first BIMF level and it shows the more highfrequency component. Figure 4 shows the reconstructed SAR images obtained for conventional Frost filter with speckle variance 0.01 and 0.05 , for three different window sizes. Figure 5 shows the reconstructed SAR images obtained for the proposed BEMD-based Frost filter.

From the visual representation of the reconstructed image, it clearly shows the appearance almost similar to the original image. Even though visual representation gives visually similar appearance for BEMD-based Frost filter. To have more accurate evaluation, we have calculated the parametric values. The performance parameter values were calculated for both reconstructed BEMD-based Frost filter for $3 \times 3,5 \times 5,7 \times 7$ window sizes of SAR image. The performance parameter values obtained for BEMD-based Frost filter are shown in Tables 1 and 2.

The performance of despeckling issues can be estimated using various parameters, and the speckle level in a SAR image can be measured by using the ENL parameter. The performance of the ENL value should be large for a good speckle noise reduction filter. The ENL values obtained for the proposed BEMD-based Frost filter with speckle variance 0.01 are $6.378,6.493$ and 6.399 for the window sizes $3 \times 3,5 \times 5$ and $7 \times 7$, respectively. When the speckle variance is 0.05 , the proposed BEMD-based Frost filter gives the ENL values as 5.558, 5.745 and 5.652, respectively. SSI parameter is used to indicate the quantity of speckle suppressed. The filter is said to be efficient for speckle noise reduction if its SSI index value does not exceed one. The SSI values obtained for the proposed BEMD-based Frost filter with speckle variance 0.01 are $0.936,0.929$ and 0.936 for the window sizes $3 \times 3,5 \times 5$ and $7 \times 7$, respectively. When the speckle variance is 0.05 , the proposed BEMD-based Frost filter gives the SSI values as $0.894,0.880$ and 0.890 , respectively. The SSIM parameter is used to quantify the similarities between two SAR images and this value should be high. The SSIM parameter value lies between zero to one. If SSIM is a greater value then it means that greater similarity exists between a reference image and despeckled image. The SSIM values obtained for the proposed BEMD-based Frost filter with speckle variance 0.01 are $0.772,0.656$ and 0.598 for the window sizes $3 \times 3,5 \times 5$ and $7 \times 7$, respectively. From the detailed experimental analysis, it is concluded that the BEMD-based Frost filtering method performance is good and the results are presented in Tables 1 and 2. We have used Matlab R2015a software to carry out all the simulation process in implementing the proposed method. We have used Matlab R2015a software to carry out all the simulation processes. An Intel 64 core i5 G50 processor with a clock speed of $2.40 \mathrm{GHz}$ and with RAM size of $4 \mathrm{~GB}$ is used for implementing the proposed work. The simulation results show that the average time taken for denoising a $256 \times 256$ size image is about $24.48 \mathrm{~s}$.

\section{Performance Parameters}

\section{Mean}

The mean value provides the pixel intensity value of the whole image, and it is represented as

$\mu=\frac{1}{N} \sum_{x=0}^{N-1} i_{x}$

where $N$ represents the number of pixels and $i_{x}$ represents the pixel intensity.

\section{Image Variance (IV)}

This represents the amount of speckle in an image. A smooth image is obtained when the image variance is less. The variance is calculated as

$\sigma^{2}=\frac{1}{N} \sum_{x=0}^{N-1}\left(i_{x}-\mu\right)^{2}$

where $\mu$ is the mean value. 
Fig. 3 BEMD decomposition. a Original SAR image,

b Speckle noise image, c BIMF-

1, d BIMF-2, e BIMF-3

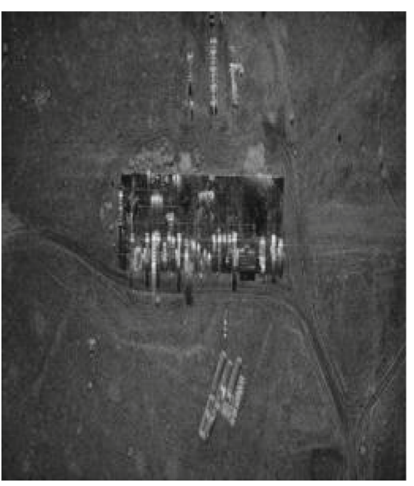

(a) Original SAR image

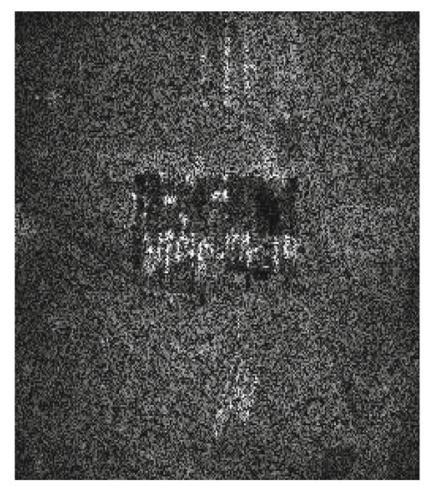

(b) Speckle noise image

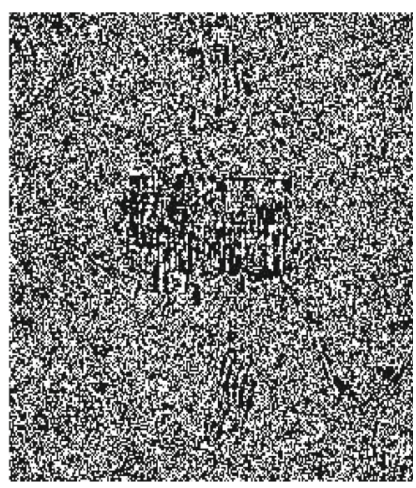

(c) BIMF-1

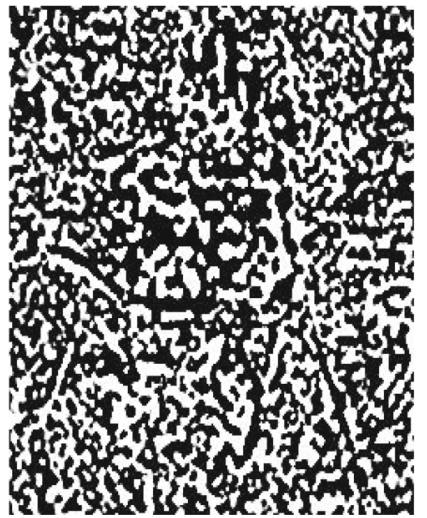

(d) BIMF-2

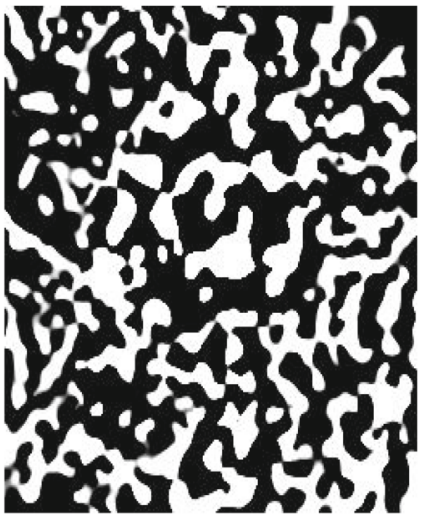

(e) BIMF-3

Fig. 4 Reconstructed SAR images using conventional Frost filter for three different window sizes. a-c with 0.01 speckle variance. $\mathbf{d}-\mathbf{f}$ with 0.05 speckle variance. a $3 \times 3$, b $5 \times 5$, c $7 \times 7$, d $3 \times 3$, e $5 \times 5$, f $7 \times 7$

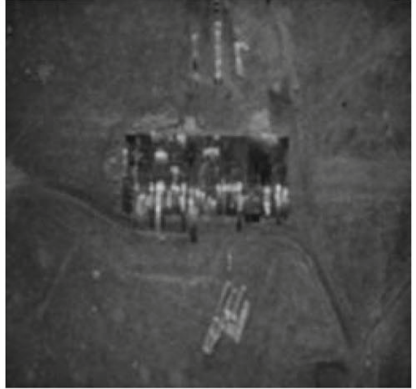

(a) $3 \times 3$

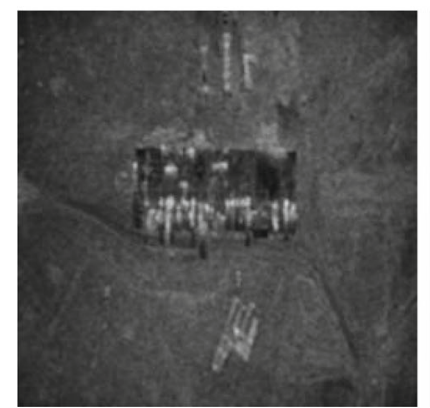

(d) $3 \times 3$

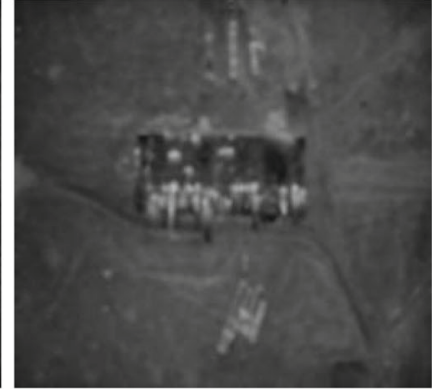

(b) $5 \times 5$

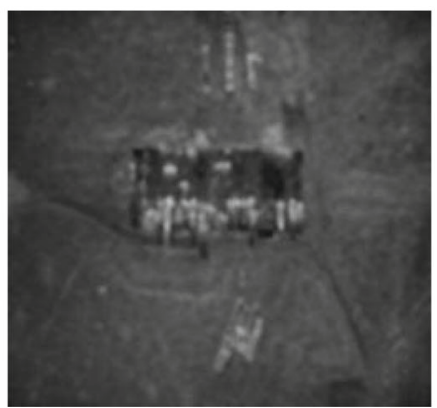

(e) $5 \times 5$

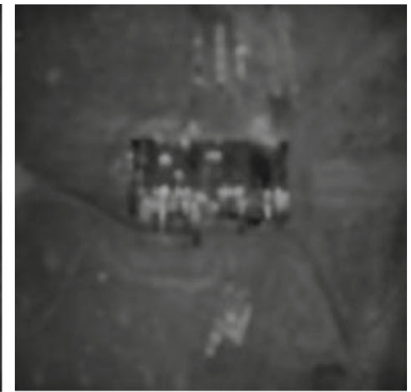

(c) $7 \times 7$

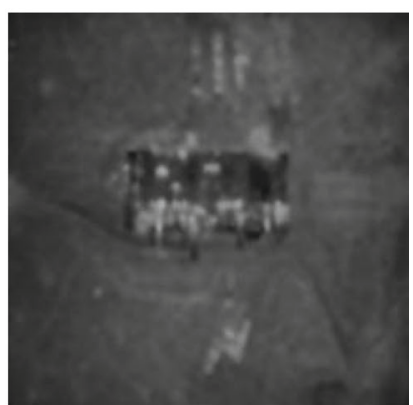

(f) $7 \times 7$

\section{Mean Squared Error (MSE)}

MSE value represents the difference of the pixel value. A higher MSE value shows that there is much difference between original and denoised image. This parameter can be calculated as 
Fig. 5 Reconstructed SAR images using the proposed BEMD-based Frost filter for three different window sizes. ac with speckle variance of 0.01 . d-f with speckle variance of 0.05 . a $3 \times 3$, b $5 \times 5$, c $7 \times 7$, d $3 \times 3$, e $5 \times 5$, f $7 \times 7$

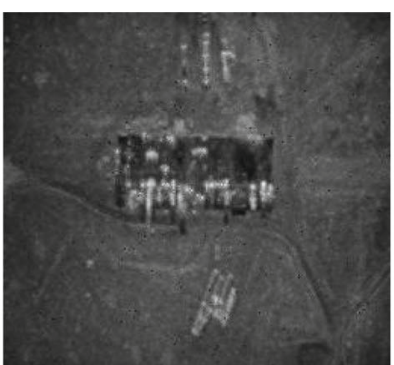

(a) $3 \times 3$

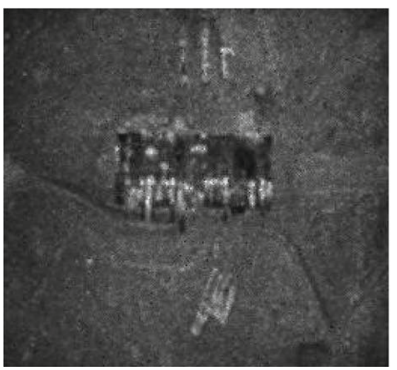

(d) $3 \times 3$

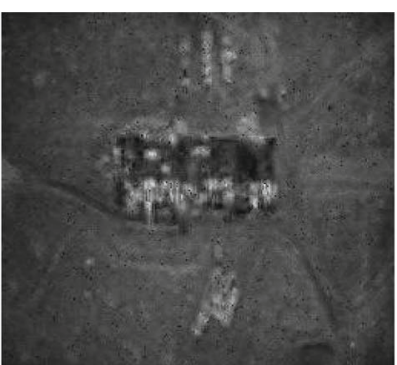

(b) $5 \times 5$

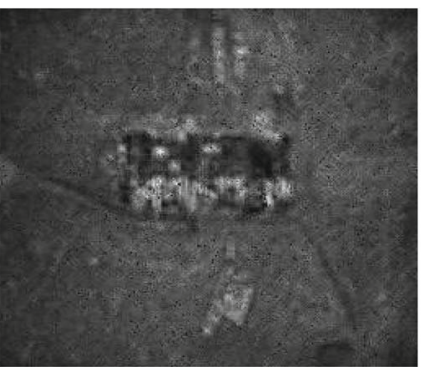

(e) $5 \times 5$

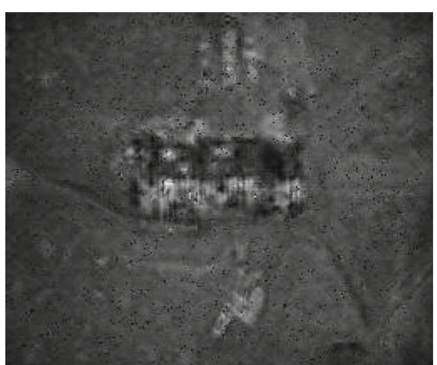

(c) $7 \times 7$

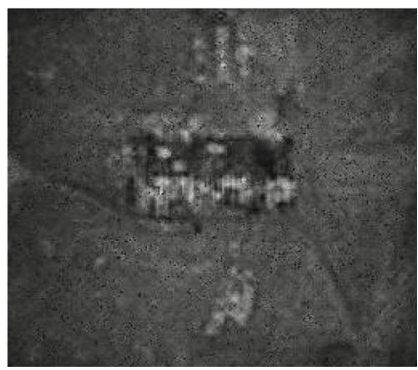

(f) $7 \times 7$

Table 1 Performance evaluation of conventional and proposed filtering methods for speckle variance 0.01

\begin{tabular}{|c|c|c|c|c|c|c|c|c|c|}
\hline Filtering method & Size of the window & MSE & PSNR & IV & Mean & SD & ENL & SSI & SSIM \\
\hline Speckled image & $3 \times 3$ & 12.892 & 37.027 & 407.75 & 47.773 & 20.193 & 5.597 & - & - \\
\hline Lee (1980) & & 17.103 & 35.800 & 299.83 & 47.771 & 17.316 & 7.611 & 0.858 & 0.707 \\
\hline Frost et al., (1982) & & 16.232 & 36.027 & 302.59 & 47.756 & 17.395 & 7.537 & 0.862 & 0.727 \\
\hline Kuan et al., (1985) & & 16.334 & 36.000 & 306.39 & 47.689 & 17.504 & 7.423 & 0.868 & 0.718 \\
\hline Lopes et al., (1990) & & 18.550 & 35.447 & 305.97 & 47.616 & 17.492 & 7.410 & 0.869 & 0.701 \\
\hline Zhu et al., (2013) & & 20.471 & 35.019 & 304.97 & 47.097 & 17.463 & 7.273 & 0.877 & 0.704 \\
\hline Proposed BEMD-based Frost & & 12.795 & 37.061 & 361.60 & 48.024 & 19.016 & 7.878 & 0.936 & 0.772 \\
\hline Speckled image & $5 \times 5$ & 12.773 & 37.068 & 411.107 & 47.804 & 20.276 & 5.559 & - & - \\
\hline Lee (1980) & & 22.663 & 34.578 & 258.829 & 47.802 & 16.088 & 8.828 & 0.794 & 0.586 \\
\hline Frost et al., (1982) & & 21.380 & 34.831 & 265.777 & 47.779 & 16.303 & 8.589 & 0.804 & 0.618 \\
\hline Kuan et al., (1985) & & 20.915 & 34.926 & 281.376 & 47.731 & 16.774 & 8.097 & 0.829 & 0.624 \\
\hline Lopes et al., (1990) & & 25.134 & 34.128 & 269.751 & 47.524 & 16.424 & 8.373 & 0.815 & 0.580 \\
\hline Zhu et al., (2013) & & 26.609 & 33.881 & 253.132 & 46.724 & 15.910 & 8.625 & 0.803 & 0.589 \\
\hline Proposed BEMD-based Frost & & 12.816 & 37.053 & 346.999 & 47.467 & 18.628 & 8.993 & 0.929 & 0.656 \\
\hline Speckled image & $7 \times 7$ & 12.844 & 37.044 & 405.988 & 47.799 & 20.149 & 5.628 & - & - \\
\hline Lee (1980) & & 25.447 & 34.074 & 230.975 & 47.793 & 15.198 & 9.589 & 0.754 & 0.532 \\
\hline Frost et al., (1982) & & 23.780 & 34.369 & 242.112 & 47.772 & 15.560 & 9.426 & 0.773 & 0.572 \\
\hline Kuan et al., (1985) & & 23.144 & 34.486 & 263.820 & 47.751 & 16.243 & 8.643 & 0.807 & 0.588 \\
\hline Lopes et al., (1990) & & 28.897 & 33.522 & 246.283 & 47.399 & 15.693 & 9.122 & 0.785 & 0.526 \\
\hline Zhu et al., (2013) & & 29.977 & 33.363 & 221.256 & 46.462 & 14.875 & 9.757 & 0.759 & 0.535 \\
\hline Proposed BEMD-based Frost & & 12.946 & 37.009 & 345.553 & 47.022 & 18.589 & 9.819 & 0.936 & 0.598 \\
\hline
\end{tabular}

$\mathrm{MSE}=\frac{1}{N} \sum_{i=0}^{N-1}\left(p_{i}-q_{i}\right)^{2}$ where $N$ represents image size, $p_{i}$ represents denoised image, and $q_{i}$ represents the original image. 
Table 2 Performance evaluation of conventional and proposed filtering methods for speckle variance 0.05

\begin{tabular}{|c|c|c|c|c|c|c|c|c|c|}
\hline Filtering method & Size of the window & MSE & PSNR & IV & Mean & SD & ENL & SSI & SSIM \\
\hline Speckled image & $3 \times 3$ & 49.035 & 31.226 & 513.304 & 47.785 & 22.656 & 4.449 & - & - \\
\hline Lee (1980) & & 21.491 & 34.808 & 310.385 & 47.785 & 17.618 & 7.357 & 0.778 & 0.512 \\
\hline Frost et al., (1982) & & 20.219 & 35.073 & 316.668 & 47.809 & 17.795 & 7.218 & 0.784 & 0.56 \\
\hline Kuan et al., (1985) & & 20.299 & 35.056 & 325.705 & 47.692 & 18.047 & 6.984 & 0.798 & 0.549 \\
\hline Lopes et al., (1990) & & 22.657 & 34.579 & 319.116 & 47.672 & 17.864 & 7.122 & 0.79 & 0.511 \\
\hline Zhu et al., (2013) & & 32.683 & 32.988 & 316.444 & 46.697 & 17.789 & 6.891 & 0.803 & 0.506 \\
\hline Proposed BEMD-based Frost & & 35.739 & 32.599 & 411.086 & 47.8 & 20.275 & 7.558 & 0.894 & 0.645 \\
\hline Speckled image & $5 \times 5$ & 49.089 & 31.221 & 512.805 & 47.803 & 22.645 & 4.456 & - & - \\
\hline Lee (1980) & & 24.071 & 34.316 & 259.869 & 47.804 & 16.12 & 8.794 & 0.712 & 0.393 \\
\hline Frost et al., (1982) & & 22.395 & 34.629 & 268.206 & 47.78 & 16.377 & 8.512 & 0.724 & 0.441 \\
\hline Kuan et al., (1985) & & 21.646 & 34.777 & 296.057 & 47.735 & 17.206 & 7.697 & 0.761 & 0.462 \\
\hline Lopes et al., (1990) & & 26.493 & 33.9 & 270.82 & 47.521 & 16.457 & 8.339 & 0.731 & 0.39 \\
\hline Zhu et al., (2013) & & 33.249 & 32.913 & 254.285 & 46.249 & 15.946 & 8.412 & 0.728 & 0.391 \\
\hline Proposed BEMD-based Frost & & 42.324 & 31.865 & 389.228 & 47.287 & 19.729 & 8.945 & 0.88 & 0.54 \\
\hline Speckled image & $7 \times 7$ & 49.01 & 31.228 & 515.929 & 47.793 & 22.714 & 4.427 & - & - \\
\hline Lee (1980) & & 26.005 & 33.98 & 235.103 & 47.784 & 15.333 & 9.712 & 0.675 & 0.346 \\
\hline Frost et al., (1982) & & 23.835 & 34.359 & 248.854 & 47.765 & 15.775 & 9.168 & 0.695 & 0.398 \\
\hline Kuan et al., (1985) & & 22.962 & 34.521 & 285.465 & 47.753 & 16.896 & 7.988 & 0.744 & 0.439 \\
\hline Lopes et al., (1990) & & 29.441 & 33.441 & 250.318 & 47.388 & 15.821 & 8.971 & 0.703 & 0.344 \\
\hline Zhu et al., (2013) & & 35.214 & 32.664 & 222.834 & 45.959 & 14.928 & 9.479 & 0.685 & 0.343 \\
\hline Proposed BEMD-based Frost & & 44.192 & 31.677 & 390.173 & 46.962 & 19.753 & 9.932 & 0.89 & 0.494 \\
\hline
\end{tabular}

\section{Equivalent Number of Looks (ENL)}

This is utilized to quantify the level of speckle noise. Improved quantization performance is obtained when ENL value is high. The formula for calculating ENL is

$\mathrm{ENL}=\left[\frac{\mu}{\sigma}\right]^{2}$.

\section{Peak Signal to Noise Ratio (PSNR)}

It evaluates the quality of a processed image compared with the denoised image. A larger PSNR value indicates a good quality image. The peak signal to noise ratio is given as

$\mathrm{PSNR}=10 \log _{10}\left(\frac{255^{2}}{\sum_{i=0}^{N-1}\left(p_{i}-q_{i}\right)^{2}}\right)$.

\section{Speckle Suppression Index (SSI)}

The fundamental goal of speckled preprocessing is to lessen the impact of random noise on value computation by suppressing image noise in homogenous regions while sacrificing boundary and texture information. SSI of a filtered image is the coefficient of its variance, which is normalized with the input image. This is formulated as

SSI $=\frac{\sqrt{\operatorname{Var}\left(I_{f}\right)}}{\operatorname{mean}\left(I_{f}\right)} X \frac{\operatorname{mean}\left(I_{o}\right)}{\sqrt{\operatorname{Var}\left(I_{o}\right)}}$

where $I_{f}$ is the filtered image, $I_{o}$ is the noisy image.

SSI value will be less than one for an efficient speckle noise reduction filter. If SSI value is lower, then the performance of the filter is better in speckle reduction.

\section{Structural Similarity Index Measure (SSIM)}

SSIM is used for measuring the similarity between original image and filtered image. A high SSIM value between the original and filtered image indicates reduced noise level and better preservation of the details.

$\operatorname{SSIM}=\frac{\left(2 \mu_{i} \mu_{j}+K_{1}\right)\left(2 \sigma_{i j}+K_{2}\right)}{\left(\mu_{i}^{2}+\mu_{j}^{2}+K_{1}\right)\left(\sigma_{i}^{2}+\sigma_{j}^{2}+K_{2}\right)}$

where $\mu_{i}$ and $\mu_{j}$ are the mean, $\sigma_{i j}$ represents the covariance of $i$ and $j, \sigma_{i}^{2}$ and $\sigma_{j}^{2}$ are the standard deviation, $K_{1}=\left(a_{1} L\right)^{2}$ and $K_{2}=\left(a_{2} L\right)^{2}$ are the two variables to balance the division with weak denominator, $L$ represents the pixel 
value range, normally it is 255 for 8-bit grayscale images and $a_{1}=0.01$ and $a_{2}=0.03$ by default.

\section{Conclusion}

This paper projects BEMD-based adaptive filtering techniques for minimizing the problem of speckle noise in SAR images. The adaptive Frost filter is used. Three BIMF levels have been used in the proposed work. Here, we have used three different window sizes like $3 \times 3,5 \times 5$ and $7 \times 7$. We have evaluated and analyzed the performance parameter values of both the filters. The proposed method will reduce the speckle in the SAR image and preserve more details compared to other methods. Frost filter with BEMD achieved better speckle reduction and retained the information. There is always trade-off between detail preservation and speckle reduction. In the future, this work can be enhanced by developing optimal adaptive filters in combination with multivariant decomposition algorithms.

Funding This research did not receive any specific Grant from funding agencies in the public, commercial or not-for-profit sectors.

\section{Declarations}

Conflict of interest The authors declare that they have no conflict of interest.

\section{References}

Achim, A., Kuruoglu, E. E., \& Zerubia, J. (2006). SAR image filtering based on the heavy-tailed Rayleigh model. IEEE Transactions on Image Processing, 15(9), 2686-2693.

Ahmed, S. H., Bouk, S. H., Javaid, N., et al. (2021). RF propagation analysis of MICAz Motes antenna with ground effect. In: S.H. Ahmed, S.H. Bouk, N. Javaid, I. Sasase (eds.) 2012 15th International multitopic conference (INMIC).

Amirmazlaghani, M., Amindavar, H., \& Moghaddamjoo, A. (2008). Speckle suppression in SAR images using the 2-D GARCH model. IEEE Transactions on Image Processing, 18(2), 250-259.

Argenti, F., \& Alparone, L. (2002). Speckle removal from SAR images in the undecimated wavelet domain. IEEE Transactions on Geoscience and Remote Sensing, 40(11), 2363-2374.

Argenti, F., Bianchi, T., \& Alparone, L. (2006). Multiresolution map despeckling of SAR images based on locally adaptive generalized Gaussian pdf modeling. IEEE Transactions on Image Processing, 15(11), 3385-3399.

Azimi-Sadjadi, M. R., \& Bannour, S. (1991). Two-dimensional adaptive block Kalman filtering of SAR imagery. IEEE Transactions on Geoscience and Remote Sensing, 29(5), 742-753.

Azimi-Sadjadi, M. R., \& Pan, H. (1994). Two-dimensional block diagonal LMS adaptive filtering. IEEE Transactions on Signal Processing, 42(9), 2420-2429.
Balamurugan, S. A., \& Kumar, S. S. (2019). IDsMA: An integrated digital signature and mutual authentication mechanism for securing the cognitive radio networks. International Journal of Communication Systems, 33(6), e4289.

Bhuiyan, M. I. H., Ahmad, M. O., \& Swamy, M. (2007). Spatially adaptive wavelet-based method using the Cauchy prior for denoising the SAR images. IEEE Transactions on Circuits and Systems for Video Technology, 17(4), 500-507.

Chen, Y., Yang, Q., Gu, Y., \& Yang, J. (2006). Detection of roads in SAR images using particle filter. In 2006 International conference on image processing (pp. 2337-2340). IEEE.

Deledalle, C.-A., Denis, L., Tabti, S., \& Tupin, F. (2017). Mulog, or how to apply Gaussian denoisers to multi-channel SAR speckle reduction? IEEE Transactions on Image Processing, 26(9), 4389-4403.

Domg, Y., Milne, A. K., et al. (2001). Toward edge sharpening: A SAR speckle filtering algorithm. IEEE Transactions on Geoscience and Remote Sensing, 39(4), 851-863.

Errico, A., Angelino, C. V., Cicala, L., Persechino, G., Ferrara, C., Lega, M., Vallario, A., Parente, C., Masi, G., Gaetano, R., et al. (2015). Detection of environmental hazards through the featurebased fusion of optical and SAR data: A case study in southern Italy. International Journal of Remote Sensing, 36(13), 3345-3367.

Everson, R. M., Roberts, S. J. (2000). Particle filters for nonstationary ICA In M. Girolami (Ed.), Advances in independent component analysis (pp. 23-41). Springer.

Feng, W., Lei, H., \& Gao, Y. (2014). Speckle reduction via higher order total variation approach. IEEE Transactions on Image Processing, 23(4), 1831-1843.

Frost, V. S., Stiles, J. A., Holtzman, J. C., Held, D. (1980). Radar image preprocessing. In LARS symposia (p. 350).

Frost, V. S., Stiles, J. A., Shanmugan, K. S., \& Holtzman, J. C. (1982). A model for radar images and its application to adaptive digital filtering of multiplicative noise. IEEE Transactions on Pattern Analysis and Machine Intelligence, 2, 157-166.

Geling, G., \& Ionescu, D. (1994). A Kalman filter for noise reduction on SAR imagery. In Proceedings of electrical and computer engineering Canadian conference (Vol. 1, pp. 405-408).

Gencaga, D., Kuruoglu, E., \& Aysin, E. (2005). Synthetic aperture radar image enhancement using particle filters. In ESA-EUSC 2005: Image information mining theory and application to earth observation.

Gleich, D., \& Datcu, M. (2009). Wavelet-based SAR image despeckling and information extraction, using particle filter. IEEE Transactions on Image Processing: A Publication of the IEEE Signal Processing Society, 18(10), 2167-2184.

Godsill, S., \& Clapp, T. (2001). Improvement strategies for Monte Carlo particle filters. In Sequential Monte Carlo methods in practice (pp. 139-158). Springer.

Habibi, A. (1972). Two-dimensional Bayesian estimate of images. Proceedings of the IEEE, 60(7), 878-883.

Hsiao, T., Rangarajan, A., \& Gindi, G. (2002). Joint-map Bayesian tomographic reconstruction with a gamma-mixture prior. IEEE Transactions on Image Processing, 11(12), 1466-1477.

Idol, T., Haack, B., \& Mahabir, R. (2015). Comparison and integration of spaceborne optical and radar data for mapping in Sudan. International Journal of Remote Sensing, 36(6), 1551-1569.

Jia, M., Zhao, Z., Huo, L., Chen, H., \& Qiu, Y. (2019). Incorporating global-local a priori knowledge into expectation-maximization for SAR image change detection. International Journal of Remote Sensing, 40(2), 734-758.

Jidesh, P., \& Balaji, B. (2018). Adaptive non-local level-set model for despeckling and deblurring of synthetic aperture radar imagery. International Journal of Remote Sensing, 39(20), 6540-6556. 
Khosravi, I., Safari, A., \& Homayouni, S. (2018). MSMD: Maximum separability and minimum dependency feature selection for cropland classification from optical and radar data. International Journal of Remote Sensing, 39(8), 2159-2176.

Kuan, D. T., Sawchuk, A. A., Strand, T. C., \& Chavel, P. (1985). Adaptive noise smoothing filter for images with signal-dependent noise. IEEE Transactions on Pattern Analysis and Machine Intelligence, 2, 165-177.

Lee, J.-S. (1980). Digital image enhancement and noise filtering by use of local statistics. IEEE Transactions on Pattern Analysis and Machine Intelligence, 2, 165-168.

Li, Y. -Q., He, M. -Y., \& Fang, X. -F. (2006). A new adaptive algorithm for despeckling SAR images based on contourlet transform. In: 2006 8th International conference on signal processing (Vol. 4). IEEE.

Lian, M., \& Jiang, Y. (2017). Time-frequency analysis for moving ship targets in geo spaceborne/airborne bistatic SAR imaging based on a geo satellite transmitter. International Journal of Remote Sensing, 38(23), 7389-7404.

Linderhed, A. (2002). 2 d empirical mode decompositions in the spirit of image compression. In Wavelet and independent component analysis applications IX (Vol. 4738, pp. 1-8). International Society for Optics and Photonics.

Liu, L., Huang, W., \& Wang, C. (2017). Texture image prior for SAR image super resolution based on total variation regularization using split Bregman iteration. International Journal of Remote Sensing, 38(20), 5673-5687.

Lopes, A., Nezry, E., Touzi, R., \& Laur, H. (1990). Maximum a posteriori speckle filtering and first order texture models in SAR images. In 10th Annual international symposium on geoscience and remote sensing (pp. 2409-2412). IEEE.

Lu, Y., Gao, Q., Sun, D., \& Zhang, D. (2014). Directionlet-based method using the Gaussian mixture prior to SAR image despeckling. International Journal of Remote Sensing, 35(3), $1143-1161$.

Metwalli, M. R., Nasr, A. H., Faragallah, O. S., El-Rabaie, E.-S.M., Abbas, A. M., Alshebeili, S. A., \& Abd El-Samie, F. E. (2014). Efficient pan-sharpening of satellite images with the contourlet transform. International Journal of Remote Sensing, 35(5), 1979-2002.

Nguyen, N. T., Leu, M. C., Zeadally, S., et al. (2018). Optimal solution for data collision avoidance in radio frequency identification networks. Internet Technology Letters, 1, e49.

Nunes, J.-C., \& Deléchelle, E. (2009). Empirical mode decomposition: Applications on signal and image processing. Advances in Adaptive Data Analysis, 1(01), 125-175.

Rajamani, A., \& Krishnaveni, V. (2014). Performance analysis survey of various SAR image despeckling techniques. International Journal of Computer Applications, 90(7), 5-17.

Ramdani, F. (2019). Recent expansion of oil palm plantation in the most eastern part of Indonesia: Feature extraction with polarimetric SAR. International Journal of Remote Sensing, 40(19), 7371-7388.
Rott, H., Yueh, S. H., Cline, D. W., Duguay, C., Essery, R., Haas, C., Heliere, F., Kern, M., Macelloni, G., Malnes, E., et al. (2010). Cold regions hydrology high-resolution observatory for snow and cold land processes. Proceedings of the IEEE, 98(5), $752-765$.

Subrahmanyam, G. R. S., Rajagopalan, A., \& Aravind, R. (2008). A recursive filter for despeckling SAR images. IEEE Transactions on Image Processing, 17(10), 1969-1974.

Sveinsson, J. R., Semar, Z., \& Benediktsson, J. A. (2008). Speckle reduction of SAR images in the bandlet domain. In IGARSS 2008-2008 IEEE international geoscience and remote sensing symposium (Vol. 3, pp. III-1158). IEEE.

Tamminen, T., \& Lampinen, J. (2006). Sequential Monte Carlo for Bayesian matching of objects with occlusions. IEEE Transactions on Pattern Analysis and Machine Intelligence, 28(6), 930-941.

Tsuchida, M., Haseyama, M., \& Kitajima, H. (2003). A Kalman filter using texture for noise reduction in SAR images. Electronics and Communications in Japan (part $i$ : Communications), 86(10), 21-32.

Van Coillie, F., Lievens, H., Joos, I., Pizurica, A., Verbeke, L., De Wulf, R., \& Verhoest, N. (2011). Training neural networks on artificially generated data: A novel approach to SAR speckle removal. International Journal of Remote Sensing, 32(12), 3405-3425.

Warner, T. A., \& Lee, J. Y. (2011). Spatial context-dependent multiscale and directional image texture. International Journal of Remote Sensing, 32(17), 4787-4806.

Woo, H., \& Yun, S. (2011). Alternating minimization algorithm for speckle reduction with a shifting technique. IEEE Transactions on Image Processing, 21(4), 1701-1714.

$\mathrm{Xu}, \mathrm{P}$. (1999). Despeckling SAR-type multiplicative noise. International Journal of Remote Sensing, 20(13), 2577-2596.

Yuan, X., Tang, T., Xiang, D., Li, Y., \& Su, Y. (2014). Target recognition in SAR imagery based on local gradient ratio pattern. International Journal of Remote Sensing, 35(3), 857-870.

Yun, S., \& Woo, H. (2012). A new multiplicative denoising variational model based on $m$ th root transformation. IEEE Transactions on Image Processing, 21(5), 2523-2533.

Zhang, X., Meng, H., Ma, Z., \& Tian, X. (2013). Sar image despeckling by combining saliency map and threshold selection. International Journal of Remote Sensing, 34(21), 7854-7873.

Zhu, J., Wen, J., \& Zhang, Y. (2013). A new algorithm for SAR image despeckling using an enhanced Lee filter and median filter. In 2013 6th International congress on image and signal processing (CISP) (Vol. 1, pp. 224-228). IEEE.

Publisher's Note Springer Nature remains neutral with regard to jurisdictional claims in published maps and institutional affiliations. 